\title{
Optimized Allocation of Resources for Intelligent Construction of Training Venues for Track and Field Teams
}

\author{
Yan Qin (i) and Wen Wu \\ Yangtze Normal University, Chongqing 408100, China \\ Correspondence should be addressed to Yan Qin; 20000022@yznu.edu.cn
}

Received 3 June 2021; Revised 24 August 2021; Accepted 31 August 2021; Published 9 September 2021

Academic Editor: Fazlullah Khan

Copyright (C) 2021 Yan Qin and Wen Wu. This is an open access article distributed under the Creative Commons Attribution License, which permits unrestricted use, distribution, and reproduction in any medium, provided the original work is properly cited.

\begin{abstract}
Because of the problems of low allocation efficiency and accuracy in the traditional resource allocation model for the intelligent construction of track and field team training venues, this paper proposes a new intelligent construction resource allocation model for track and field team training venues. A distributed data collection method is adopted to select the index data for the intelligent construction of the track and field team training venues to optimize the allocation of resources and the mined resource allocation index through extracting the optimized index characteristics. The goal of the optimization problem is transformed into a singleobjective solving problem. The suggested approach is based on the two-level programming concept. The simulation experimental results show higher efficiency and better allocation effect of the proposed scheme compared to other state-of-the-art approaches. Our evaluation and observations suggest that the accuracy of the intelligent construction of track and field team training venues is between $90 \%$ and $99 \%$ which is higher than two other benchmarked models. Furthermore, the proposed model can quickly reach to an optimal decision.
\end{abstract}

\section{Introduction}

According to a special survey of the national track and field team coaches, athletes, scientific research managers, related auxiliary staff, etc., the demand for intelligent construction of the national track and field team training venues basically has reached up to approximately 99\%. China has conducted several comprehensive surveys of track and field team training venues many times. After the fourth census, it was discovered that there have been great changes in the number and scale of track and field team training venues, and many more training venues have appeared. The comprehensive track and field team training venues that are in line with the sports spirit of the current era have brought hope for the development of sports in China [1]. However, with the passage of time, the requirements for track and field team training venues have constantly increased. This also needs the track and field team training venues to have many functions. Moreover, current sports are gradually becoming more diversified, which makes the track and field team training venues more demanding. Various sports venues and various track and field team training facilities will essentially make the management department to complete more complicated tasks when managing the resources when it is impossible to use advanced equipment under certain conditions.

With management methods, not only there will be errors in sports venues, but this will also result in serious waste of resources for intelligent construction of track and field training venues [2]. How can we make better use of the intelligent construction resources of the track and field team training venues, under the premise of (i) ensuring the scientific management of the track and field team training venues as much as possible and (ii) achieving rapid management of related information, is the focus of modern research in sport-related issues [3]. Track and field training venues are of great significance to holding large-scale competitions and will play a very important role in the development of sports activities. However, because of many issues and reasons, the current level of information 
management of track and field training venues is not high enough. At the same time, at present, many of our country's track and field team training venues still have great shortcomings in terms of information technology. In order to improve (a) the track and field team training venues, (b) informatization construction, (c) response to the spirit of popular sports, and (d) the basic comprehensive construction of the track and field team training venues, many researchers have studied the intelligent construction resource optimization models and have obtained acceptable results. For example, in [4], the authors constructed a digital construction resource optimal allocation ecosystem model, which can not only straighten out the relationship between the allocation subject and the ecological environment (cooperation, competition, and adaptation) but also reveal the operating mechanism of the ecosystem (resources). Besides circulation, collaborative competition, mutual symbiosis, dynamic balance, and value appreciation, the authors also highlight the principles of continuous "evolution" for digital construction resources in the allocation ecosystems.

The construction of an ecosystem model for the optimal allocation of digital construction resources is conducive to promoting China's digital construction resources. The configuration has evolved from a state of "disordered, inefficient, and unstable" to a state of "ordered, efficient, and stable," providing a useful reference for China's digital resource construction practice. The authors in [5] constructed a model for the optimal allocation of stadium resources from the perspective of public sports services. The suggested model comprehensively uses the literature and combines the data method and logical analysis method (i) to clarify the values of the optimization of stadium resources, (ii) to improve the level of sports public services, and (iii) to better utilize the aims at the venues existing in the resource allocation of sport stadiums. Considering real-world problems such as unscientific resource layout, unsound operating system, and inadequate organization of public sports activities, the authors suggest that comprehensively optimizing the layout of the stadium resources could create a smart service operation management system to carry out fitness activities with characteristics of the masses, improve stadium resources utilization efficiency, promote the equalization of basic public sports services, and scientifically allocate stadium resources. However, the accuracy and efficiency of the abovementioned model for optimal allocation of the intelligent construction resources are relatively unexplored.

This paper is focused on scientifically promoting the intelligent construction of training venues for the national track and field team and realizing the main purposes of intelligent venues in science and technology. This will help in preparing appropriate tools for the Olympics, big data collection of excellent athletes' comprehensive athletic ability, intelligent information comprehensive management, cloud sharing, etc., in view of the existing models. This article builds a resource optimization allocation model for the aforementioned problem and verifies the effectiveness of the proposed model through simulation experiments. Besides, we also offer a theoretical basis and scientific support for the physical construction of the intelligent track and field team training venues. The gaps in the functional design of the construction of chemical training venues are identified. The following are the major contributions of the research conducted in this paper:

(i) Builds a resource optimization allocation model for the intelligent construction of track and field team training venues

(ii) Verifies the effectiveness of this model through simulation experiments

(iii) Provides theoretical basis and scientific support for the physical construction of the intelligent track and field team training venues

(iv) Fills up the intelligence at home and abroad

The structure of the remaining part of this paper is as follows. Section 2 illustrates the intelligent resource allocation problem. Section 3 describes the proposed research methodology. An analytical model is also presented. In Section 4, we evaluate the proposed model through experiments and discuss the results. Finally, Section 5 concludes this paper along with directions for further research and investigation.

\section{Resource Allocation Problem}

The allocation of resources for the intelligent construction of track and field team training venues refers to the problem of how people reasonably allocate resources among time, space, and departments during track and field team training activities. Moreover, this also refers to how to make sure that the scarce track and field team training venues' intelligent construction resources get the most adequate and maximum resources. The objective is to make an effective and most reasonable use of resources to meet various sport-related needs to the maximum extent [6]. The so-called method of allocation of resources for the intelligent construction of track and field team training venues refers to the form of allocation of resources for the intelligent construction of track and field team training venues. To study the allocation method of the intelligent construction resources of the track and field team training venues, we must first analyze the fundamental factors that determine the resource allocation method. The resource allocation method adopted for the intelligent construction of track and field team training venues is generally restricted by the economics of the systems. For example, those countries and regions that implement a planned economic system generally adopt an appropriate planned allocation method, while those countries that implement a market economic system generally adopt the market allocation method, as illustrated in [7].

Through the analysis of the determinants of the resource allocation method for the intelligent construction of the track and field team training venues, it can be considered that the resource allocation method for the intelligent construction of the track and field team 
training venues can be understood from the meaning of the word. Although it refers to the intelligent construction resource allocation of the track and field team training venues, but from the origin, it can be considered that the allocation of resources for the intelligent construction of track and field team training venues refers to the basic system for the allocation of resources for the intelligent construction of track and field team training venues [8]. The resource allocation method for the intelligent construction of track and field training venues is determined by the economic system, as described earlier. In addition to the abovementioned two ways of configuring resources for the intelligent construction of track and field team training venues, there is also a hybrid configuration way that integrates the abovementioned two ways of configuration. Therefore, in terms of types, there are three ways to allocate resources for the intelligent construction of track and field training venues: (i) planned allocation, (ii) market allocation, and (iii) planned and market hybrid allocation [9]. In respect of (iii), it combines the benefits of both (i) and (ii).

\section{Optimized Allocation of Resources for Intelligent Construction of Training Venues for Track and Field Teams}

In this section, we briefly describe and mathematically model the intelligent resource allocation problem as an optimization problem. First, mining the indexes, optimizing them, and feature extraction are discussed as parts of the proposed methodology. Second, an intelligent resource allocation model is proposed.

3.1. Mining Indexes for Optimal Allocation of Resources. Based on the resource allocation analysis for the intelligent construction of the track and field team training venues, the indicators for the optimal allocation of resources are evaluated. Scientific and accurate selection of allocation indicators is the prerequisite and basis for analyzing the efficiency of the resource allocation in the intelligent construction of track and field training venues. Generally, the selection of allocation indicators should follow the principles of "homotropy," "scientific," and "reasonableness." From the analysis of the actual situation of the allocation of resources, the input of the resource elements for the intelligent construction of the track and field team training venues mainly comes from three aspects: (i) the government, (ii) the unit, and (iii) the society. The intelligent construction of the track and field team training venues mainly includes service time, cost, and quality. Therefore, this study also chooses these three data items, including comprehensive service time, service cost, and service quality, as indicators and evaluation metrics to measure the optimal allocation of resources for the intelligent construction of track and field team training venues.

Before constructing the optimal allocation of resources for the intelligent construction of track and field team training venues, it is necessary to dig out indicators to measure the optimal allocation of resources for the intelligent construction of track and field team training venues [10]. To improve the comprehensiveness of the data collection, the distributed data collection method is adopted, and the index data of the intelligent construction resource optimization configuration of the track and field team training venues are selected for mining and correlation analysis. Because of duplicate and potentially wrong data in the dataset, the data are cleaned and converted using hierarchical reduction classification cleaning strategy. This method is also used to measure dirty data, and the calculation formula is given as follows:

$$
M=\frac{s^{\prime \prime}}{\sum_{a} \mathrm{~d} n} .
$$

In formula (1), $M$ represents the preprocessing result of the index data for the optimal allocation of intelligent construction resources for the track and field team, $\sum_{a} d$ represents the dirty data in the index data for the optimal allocation of the intelligent construction resources for the track and field team, and $n$ represents the amount of index data for the optimal allocation of resources for intelligent construction of training venues, and $s^{\prime \prime}$ stands for the data uniform format parameter [11]. Since these data come from the database, the dataset is divided according to the distributions of the data nodes. The calculation formula is given as follows:

$$
G=\frac{g}{p} * n^{\prime \prime \prime} M .
$$

In formula (2), $G$ represents the data characteristics of each data node, $p$ represents the attribute space of the node data, $g$ represents the division parameter, and $n^{\prime \prime \prime}$ represents the division object. Note that $M$ is computed using equation (1).

On this basis, time series are used to mine the index objects of the intelligent construction resource optimization configuration of the track and field team training venues. The purpose is to avoid the text similarity due to the complexity of the time-type data mining objects, including sequence rules, periodic patterns, and sequences mode [12]. Therefore, to reduce the calculation time, we try to find out the frequent item sets in the index data for the optimal allocation of the intelligent construction resources of the track and field team training venues and generate strong association rules based on the frequent item sets in these data. Finally, the data are being mined in the database. The mining algorithm is based on time series and is given as follows:

$$
h|f|=\sum_{i=1} e G * \frac{i}{n} .
$$

In formula (3), represents the time series parameter of the data, $e$ represents the relevance of the data, and $i / n$ represents the frequent item sets of the data. Note that $G$ is computed using equation (2).

According to the above process, data mining process of the intelligent construction resource optimization configuration index data of the track and field team training venues 
is completed based on distributed data collection method. The mined data are finally integrated into a unified file, and the cached data can be synchronized with the web version through desktop operations. It should be noted that we adopt the centralized processing and sharing of resources approaches for the intelligent construction of the track and field team training venues under various operations and provide a basis for the optimization of indicators for the allocation of resources.

3.2. Resource Allocation Index Optimization. In this section, we optimize the resource allocation indicators for the intelligent construction of track and field team training venues as mentioned above. Regarding the platform operator as the upper-level decision-maker and taking the strongest service sustainability as its objective function, the comprehensive knowledge contribution $\mathrm{K}$, service evaluation $E$, and resource use priority $P$ can represent their interests as a whole, as described in [13]. The objective functions of service sustainability index optimization are as follows:

(1) The greatest contribution to knowledge:

$$
\max K=\sum_{i=1}^{m} \sum_{k i=1}^{n i} x_{i k i} K_{c}\left(i k_{i}\right) .
$$

(2) Best service evaluation:

$$
\max E=\sum_{i=1}^{m} \sum_{k i=1}^{n i} x_{i k i} E_{v a}\left(i k_{i}\right) .
$$

Regarding the service demander as the lowerlevel decision-maker and optimal Quality of Service (QoS) as its objective function, the comprehensive service time $T$, service cost C, and service quality $Q$ can represent their interests as a whole [14]. The objective functions of the QoS index optimization problem are as follows:

(1) Shortest service time:

$$
\min T=T_{m a}+T_{t r}
$$

(2) Lowest service cost:

$$
\min C=C_{m a}+C_{t r}
$$

(3) Best service quality:

$$
\min Q=1-Q_{r a}
$$

3.3. Index Feature Extraction. Based on the abovementioned optimized resource allocation index for the intelligent construction of track and field team training venues, the index characteristics are extracted. The defined feature model slice is a unary operation that acts on feature model $W$. The input of this operation is called the slicing criterion, that is, the user's demand for feature selection [15]. The slicing criterion is composed of the required features set and the removed features set specified by the user. The output result is called the slice results set, which is composed of the dependent features set and the repelling features set, as illustrated in [15]. There are the following three constraints on the intelligent construction resource allocation model of the track and field team training venues:

$$
\begin{gathered}
\eta_{\text {in }}=\left\{\cap x \mid x \in h_{s l}\right\}, \\
\eta_{\text {excel }}=\left\{\cap\left(\frac{H}{y}\right) \mid y \in h_{s l}\right\} .
\end{gathered}
$$

Among them,

$$
h_{s l}=\left\{z \mid z \in W \wedge h_{s l} \subseteq z\right\} .
$$

In the formula, $h_{s l}$ represents the intelligent construction resources of all track and field team training venues that meet the slicing criteria, $H$ represents the limited features set in the resource allocation of the intelligent construction of the track and field team training venues, $\eta_{\text {in }}$ represents the dependent features set, and $\eta_{\text {excel }}$ represents the repelling features set. Moreover, $x, y$, and $z$ represent conventional variables, respectively. The above constraints are derived from the semantics of the feature model, and the slice results set $\prod_{s l}(W)=\left\langle\eta_{i n}, \eta_{\text {excel }}\right\rangle$ is obtained. The result of feature slicing reflects the feature set most closely related to the specific needs of users, and based on this, the optimization of resource allocation for the intelligent construction of track and field team training venues is further realized.

The calculation of the feature slice results set needs to meet the user needs and the overall constraint information of the feature model. A calculation model is designed to calculate the slice results set. The input of the algorithm is the user's choice of features, and finally, for the optimized configuration of the track and field team training stadium intelligence, the features set $N_{\text {in }}$ and the repelling features set $N_{s l}$ contained in the chemical construction resources are outputted as the dependent features set $P_{\text {in }}$ and the repelling features set $P_{s l}$, respectively.

We first traverse each feature in the $N_{\text {in }}$ set. The specific operation is as follows: if the feature does not belong to the feature-dependent set $P_{\text {in }}$ and the feature-excluded set $P_{s l}$, then it will be merged into the set $P_{i n}$. At the same time, starting from the feature node, search for nodes related to the feature in the other two directions. One of the directions is toward the parent node until the root node is found, and the other direction is toward the child node until the terminal node is found. Any feature node in the two directions will be included in the feature dependency set if it satisfies the dependency relationship. In the same way, the algorithm traverses every feature in the set $N_{s l}$, and the traversal process is still carried out from two directions. The difference is that the latter one only finds its parent node when searching in the direction of its parent node. Then, it only includes its parent node's characteristics when the characteristic node is an And-Group decomposition relationship that satisfies the required attributes. Similarly, the search in the direction of the child node is also the same as the feature dependency set until the terminal node is searched and found. 
According to the above search process and proposed algorithm, after the optimization phase is completed, the resource allocation index characteristics of the intelligent construction of the track and field team training venues are extracted in order to provide and offer a basis for the design of the intelligent construction resource allocation model of the track and field team training venues of the sports.

3.4. Construction of Resource Optimization Allocation Model. After extracting the optimized resource allocation index for the intelligent construction of track and field team training venues, the multiobjective optimization problem is transformed into a single-objective problem and solved using the minimax method. This method is preferred as it is simple and can minimize all the objectives simultaneously. It is also possible to solve each objective of the multiobjective optimization problem individually and then feed the outcome of one objective as input for the other objective and so on. However, the latter approach is complex and needs significant efforts. Furthermore, the resource optimization allocation model for the intelligent construction of track and field team training venues is based on two-level programming. The single objective is expressed as follows by equation (12) and the constraints of the problem are given by equation (13):

$$
\begin{aligned}
& P_{1} \max S=\min \left\{\frac{K}{K^{\prime}}, \frac{E}{E^{\prime}}\right\}, \\
& \text { s.t. } K_{c}(i) \geq K_{\min }, \quad i=1,2, \ldots, m, E_{v a}(i) \geq E_{\min }, \quad i=1,2, \ldots, m, \\
& P_{2} \min Q o S=\max \left\{\frac{T}{T^{\prime}}, \frac{C}{C^{\prime}}, \frac{Q}{Q^{\prime}}\right\}, \\
& \text { s.t. } T \leq T_{\max }, \\
& C \leq C_{\max }, \quad \\
& Q_{r a}(i) \geq Q_{\min }, \quad i=1,2, \ldots, m, \\
& \sum_{k i}^{n i} x_{i k i}=\left\{\begin{array}{l}
1, \text { the } i-\text { th subtask is completed by candidate resource } k_{i}, \\
0, \text { other. }
\end{array}\right.
\end{aligned}
$$

Among them, the calculated values of $K^{\prime}, E^{\prime}, T^{\prime}, C^{\prime}$, and $Q^{\prime}$ represent the maximum value of $K, E, T, C$, and $Q$. Note that the values of $K_{c}(i)$ and $E_{v a}(i)$ cannot be lower than the minimum knowledge contribution $K_{\min }$ required by the platform operator and the minimum service evaluation $K_{\min }, T, C$ and should not exceed the service demander's requirements. Similarly, the maximum service time $T_{\max }$ and the maximum cost $C_{\max }, Q_{r a}(i)$ should not be lower than the minimum quality $Q_{\min }$ required by the service demander.

In the above model, the low-level decision-makers feedback their optimal decisions to the upper-level decisionmakers under the upper-level parameter conditions. Later, the upper-level decision-makers make optimal decisions on the input's basis from low-level decision-makers. Finally, the upper-level decision-makers combine both outcomes for the use of intelligent construction resources in the track and field team training venues. The algorithm finally determines the optimal solution of the proposed model.

\section{Simulations and Experimental Outcomes}

To verify the effectiveness of the intelligent construction resource optimization model for track and field team training venues constructed in this paper in practical applications, a parallel experimental platform, based on Hadoop, was built for simulation experiments. The process of building a Hadoop distributed environment enabled us to simulate the Linux open-source environment under the Windows operating system through Cygwin64. Other essential tools, such as jdk64 and Tomcat server, were installed and ready to start building Hadoop platform. Three nodes, i.e., a master and two slave nodes, were interconnected. We set the username on the server side as administrator, a master node, and two slave nodes; the deployment work is performed on the master branch, and slave1 and slave 2 are configured as client nodes. Modifying the hosts on the master host is mainly to change the calculation and the path in the system to /in the etc./hosts file, adding the IP addresses of the master node and the slave nodes at the end of the hosts' file. We installed and started the SSH service, configured the SSH password-free login function, modified the Hadoop configuration file, and added the JDK installation path to the Hadoop-env.xml file. To run the experiment, we entered the "start-all.sh" start command to start Hadoop system. The experimental platform was designed through appropriate networking tools.

After the construction of the experimental platform is completed, the intelligent construction resource optimization allocation model was implemented. Moreover, two 


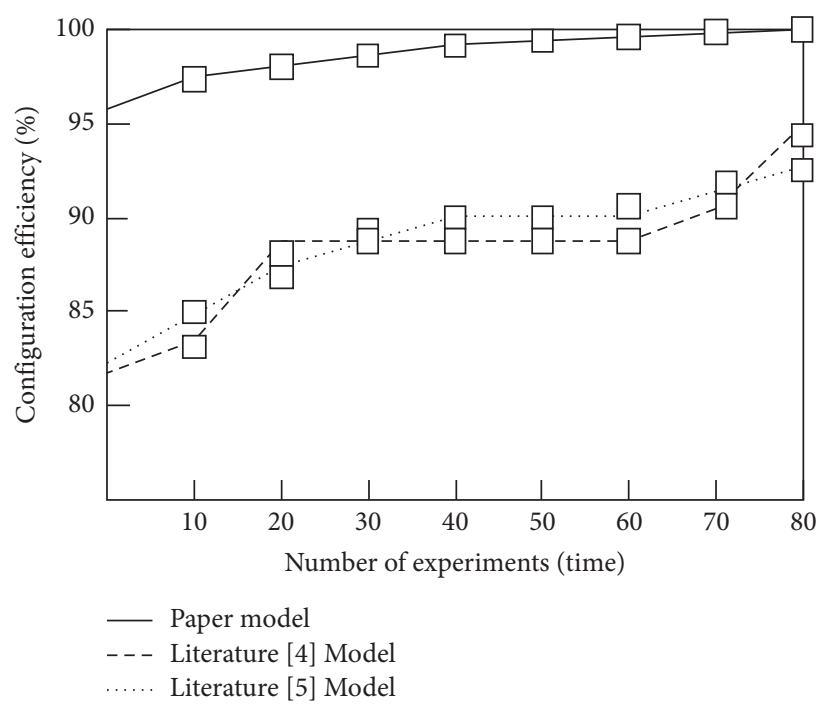

Figure 1: Comparison results of the configuration efficiency of the three models.

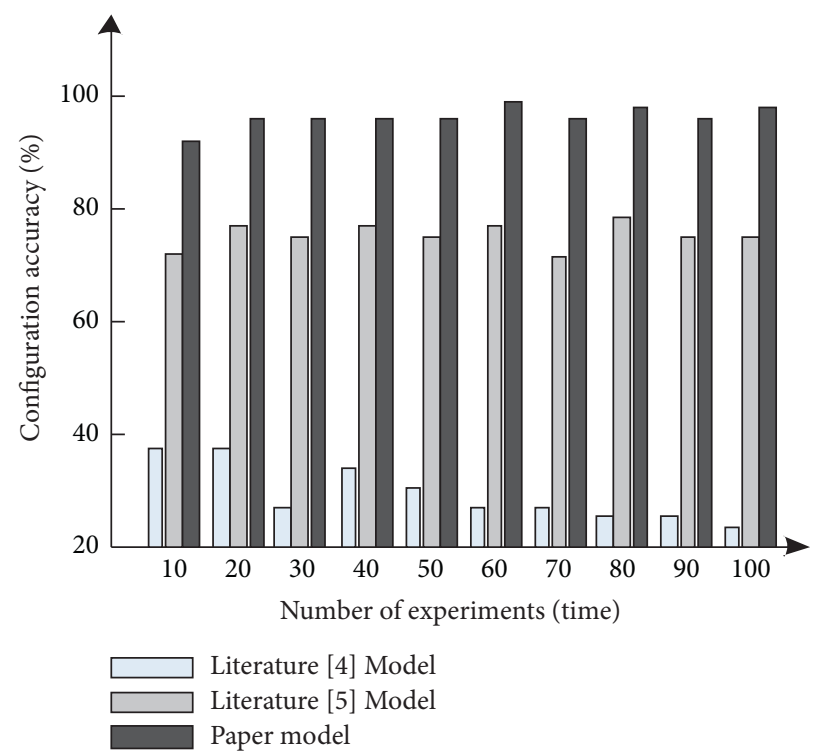

FIGURE 2: Comparison results of the configuration accuracy of the three models.

state-of-the-art models, as suggested in $[4,5]$, of the track and field team training venues were reconstructed in this paper to compare and analyze the efficiency of the proposed intelligent construction resource optimization configuration of the track and field team training venues. The comparison results, in terms of the configuration efficiency, are shown in Figure 1 . The experiments were run several times, and the outcomes represent the mean values.

According to Figure 1, it can be observed that the intelligent construction of track and field team training venues based on the intelligent construction resource allocation model of track and field team training venues constructed in this paper can reach up to $100 \%$ efficiency. For the intelligent construction of track and field team training venues based on the document [4] model, the highest resource optimization allocation efficiency is only $94 \%$, while the resource optimization allocation efficiency for reference [5] model of the track and field team training stadium intelligent construction is only $92 \%$. This demonstrates that the track and field team constructed in this paper is more efficient and can reach quickly reach to optimal solution for the track and field team intelligent resource allocation compared to other baseline approaches. The configuration efficiency of optimal allocation of resources for intelligent construction of training venues is higher than that of the models suggested in $[4,5]$.

To further verify the effectiveness of the model proposed in this paper, the intelligent construction resource optimization allocation model of the track and field team training venues, other state-of-the-art models $[4,5]$, and their resource optimization allocation accuracy metrics were analyzed and investigated. The comparison results and 
outcomes are shown in Figure 2. For baseline, benchmarked, intelligent resource allocation models, interested readers should refer to $[4,5]$.

According to Figure 2, we can see that, for the model of intelligent construction of track and field team training venues constructed in this paper, the resource optimization allocation accuracy is always between $90 \%$ and $100 \%$, while for the track and field team training venues of the model in [4], the accuracy of the intelligent construction resource optimization is between $20 \%$ and $40 \%$. Similarly, the intelligent construction resource optimization model accuracy of the track and field team training venues in the model in [5] is between $70 \%$ and $80 \%$. Compared to these baseline models, the average accuracy of the model for the intelligent construction of track and field team training venues constructed in this paper is approximately between $70 \%$ and $80 \%$. Therefore, the intelligent construction resource optimization allocation accuracy of the track and field team training venues based on the model of optimized construction resource allocation is higher than the allocation accuracy of models suggested in $[4,5]$.

\section{Conclusions and Future Work}

Looking at the overall situation of the resource allocation for intelligent construction of training venues for track and field teams in our country, i.e., China, their efficiency for most track and field teams is relatively unexplored. Moreover, the efficiency of the resource allocation construction models considerably varies across various provinces and cities. The differences in regional economic development levels, population densities, and geographic areas affect the efficiency of resource allocation for the intelligent construction of track and field team training venues to a certain extent. However, the ineffective scale of resource allocation and poor resource management status are the key cause of resource wastages. This may also be the main reason for low configuration efficiency. Based on these situations, it is recommended to incorporate the optimal allocation of resources for the intelligent construction of track and field team training venues into the regional, urban, and rural construction plan. Moreover, our outcomes demonstrate the following: (a) formulating a local track and field team training venue investment policy based on the allocation of resources for the intelligent construction of track and field team training venues, (b) adjusting the scale of resource allocation and optimizing resource allocation structure, (c) improving the efficiency of resource allocation, and (d) incorporating the optimal allocation of resources for the intelligent construction of track and field team training venues into the regional economic development plan. These steps will essentially improve the venue capital guarantee and supervision system, increase capital investment, encourage market participation, clarify management systems, and improve capital utilization efficiency. In the future, we intend to incorporate the optimal allocation of resources for the intelligent construction of track and field team training venues into the regional work performance evaluation, to improve the evaluation, supervision, and management of the optimal allocation of resources, and effectively guide the steady development of the training venues. We also intend to study the behavior of the proposed model across different geographical cities while using different datasets in the evaluation. We will also generalize the proposed model findings and compare it with other state-of-the-art methods in the field of sports. We will also investigate the machine learning approaches for intelligent resource allocation [16].

\section{Data Availability}

The data used to support the findings of this study are available from the corresponding author upon request.

\section{Conflicts of Interest}

The authors declare that they have no conflicts of interest.

\section{Acknowledgments}

This work was supported by the Chongqing Educational Science Planning Project (No. 2016-GX-148).

\section{References}

[1] I. M. Shohet, M. Luzi, and M. Tarshish, "Optimal allocation of resources in construction safety: analytical-empirical model," Safety Science, vol. 104, pp. 231-238, 2018.

[2] B. Yang, "Research on the laboratory management mode based on the optimal allocation of resources," Open Access Library Journal, vol. 08, no. 1, pp. 1-8, 2021.

[3] S. Mishra, M. N. Sahoo, A. K. Sangaiah, and B. Sambit, "Nature-inspired cost optimisation for enterprise cloud systems using joint allocation of resources," Enterprise Information Systems, vol. 15, pp. 1-23, 2019.

[4] W. Yang, J. Xu, and H. Li, "Construction of an optimal allocation model of digital education resources from the perspective of ecology," Modern Distance Education Research, vol. 2, pp. 94-102, 2018.

[5] R. Yun, "Research on optimal allocation of stadium resources from the perspective of public sports services," Journal of Guangzhou Sport University, vol. 38, no. 05, pp. 53-55, 2018.

[6] Jiayang, Y. Chun, M. Xiaotian, G. Yachun, F. Chuanji, and Y. Hongchun, "Suppressing epidemic spreading by optimizing the allocation of resources between prevention and treatment," Chaos, vol. 29, no. 11, Article ID 113108, 2019.

[7] F. Zhang, "The inspiration of thel"Eight pairs)"Countermeasures of the new supply to the optimal allocation of educational resources in universities," Research and Practice of Innovation and Entrepreneurship Theory, vol. 001, no. 007, pp. 59-60, 2018.

[8] R. Zheng, M. Chen, and Y. Zhang, "Research on optimal allocation of land resources decision-making based on MAS and PSO algorithms," Modern Management, vol. 008, no. 002, pp. 192-204, 2018.

[9] Z. Guo and G. Chen, "Predefined-time distributed optimal allocation of resources: a time-base generator scheme," IEEE Transactions on Systems, Man, and Cybernetics: Systems, vol. 99, pp. 1-10, 2020.

[10] X. Huang, H. Li, X. Zhang, and X. Zhang, "Land use policy as an instrument of rural resilience - the case of land withdrawal mechanism for rural homesteads in China," Ecological Indicators, vol. 87, pp. 47-55, 2018. 
[11] W. A. Gang, Z. Geng, G. A. Xin, and Y. Zhang, "Digital twindriven service model and optimal allocation of manufacturing resources in shared manufacturing," Journal of Manufacturing Systems, vol. 59, pp. 165-179, 2021.

[12] T. Halabi, M. Bellaiche, and A. Abusitta, "Online allocation of cloud resources based on security satisfaction," in Proceedings of the 2018 17th IEEE International Conference On Trust, Security And Privacy In Computing And Communications, pp. 379-384, New York, NY, USA, August 2018.

[13] X. Huang, W. S. Cheng, and L. M. Jiao, "Measurement and spatial heterogeneity of land use eco-efficiency and the optimal allocation of land resources," Chinese Journal of Ecology, vol. 37, no. 12, pp. 3809-3816, 2018.

[14] X. Wang, Y. Qin, W. Yang, and S. Yuan, "Fuzzy programming method for multi-objective optimal allocation of sediment resources and the cooperative bargaining: a case study in Weishan irrigation area, China," Environmental Science and Pollution Research, vol. 27, no. 8, pp. 1-16, 2020.

[15] A. Xw, Q. A. Ying, B. Wy, and K. S. China, "Multi-objective optimal allocation of sediment resources based on the subjective trade-off rate method," Journal of Cleaner Production, vol. 234, pp. 1059-1071, 2019.

[16] A. Ali, Y. Zhu, and M. Zakarya, "A data aggregation based approach to exploit dynamic spatio-temporal correlations for citywide crowd flows prediction in fog computing," Multimedia Tools and Applications, vol. 11, pp. 1-33, 2021. 